\title{
Directional detection of Dark Matter with nuclear emulsion based detector
}

\author{
Nicola D'Ambrosio ${ }^{1}$ on behalf of the NEWSdm Collaboration \\ LNGS-INFN Laboratori Nazionali del Gran Sasso \\ Via G. Acitelli - Assergi (AQ), ITALY \\ E-mail: nicola.dambrosio@lngs.infn.it
}

Direct dark matter searches are promising techniques to identify the nature of dark matter particles. A variety of experiments have been developed over the past decades, aiming to detect Weakly Interactive Massive Particles (WIMPs) via their scattering in a detector medium. Exploiting directionality would also give a proof of the galactic origin of dark matter making it possible to have a clear and unambiguous signal to background separation. The directional detection of Dark Matter requires very sensitive experiments combined with high performance technology. The NEWSdm experiment, based on nuclear emulsions, is proposed to measure the direction of WIMP-induced nuclear recoils. We discuss the potentiality, both in terms of exclusion limits and potential discovery, of a directional experiment based on the use of a solid target made by newly developed nuclear emulsions and read-out systems reaching submicrometric resolution

EPS-HEP2017, European Physical Society conference on High Energy Physics 5-12 July 2017

Venice, Italy

\footnotetext{
${ }^{1}$ Speaker

(c) Copyright owned by the author(s) under the terms of the Creative Commons

Attribution-NonCommercial-NoDerivatives 4.0 International License (CC BY-NC-ND 4.0).
} 


\section{Introduction}

The evidence for the existence of Dark Matter (DM) comes from the observation that various luminous objectsmove in the galaxies move faster than they would do if they only felt the gravitational attraction of visible matter. The existence of DM requires new particles beyond the standard model of particle physics.

Current experimental effort in the field of direct Dark Matter (DM) searches are devoted to the search for rare interactions between galactic halo Weakly Interactive Massive Paricles (WIMPs) and nuclei in a detector on Earth. As dark matter detectors are rapidly improving in sensitivity they will encounter the neutrino background, at which point Solar, atmospheric, and diffuse supernova neutrinos can mimic the dark matter signal.

Neutrino interactions are an irreducible source of background, they cannot be shielded against and produce recoil with similar rates and energy spectra, however directional detection can still provide an unambiguous observation of dark matter interactions. A new generation detectors capable of measuring the direction of a nuclear recoil track resulting from the elastic scattering of a target nucleus by incoming WIMP would provide a route towards the discrimination of neutrino background and the unambiguous identification of WIMPs as being responsible for the galactic dark matter.

The more diffuse experimental strategy is based on the use of low pressure gaseous Time Projection Chambers (TPCs) [1]. Experiments based on this technology have provided the first directional limits in the Spin-Dependent (SD) case and the collaborations aim at developing larger detectors to be operated in a medium term time scale, nevertheless this technology is hardly scalable to very large detectors masses needed to reach a good sensitivity to the SpinIndependent (SI) case. TPCs technology is hardly scalable to very large detectors masses needed to reach a good sensitivity to the Spin-Independed (SI) case.

The use of a solid target for directional searches would overcome the mass limitation of gaseous TPC approach thus allowing to reach a high sensitivity in the low cross section sectors of the SI case. Nevertheless, in a solid medium, the track of the WIMP-scattered nuclear recoil will have a path length of the order of a few hundred nanometers, much shorter than in the case of a gaseous target where the recoil length is expected to be of the order of a few millimeters. A detector with high tracking resolution is therefore needed.

\section{The NEWSdm experiment}

The approach proposed by the NEWSdm Collaboration [2] consists in the use of a nuclear emulsion-based detector acting both as target and as tracking device. The detector will use the new generation of nuclear emulsion called Nano Imaging Trackers (NIT) and UltraNano Imaging Trackers (U-NIT) $[3,4]$. The position resolution of these new nuclear emulsion is one order of magnitude higher than that of the emulsion used in the OPERA experiment [5].

The detector will be basically a bulk of NIT surrounded by a shield to reduce the external background. The detector will be placed on an equatorial telescope in order to compensate the Earth rotation, keeping fixed its orientation with respect to the incoming apparent WIMP flux. The angular distribution of the WIMP scattered nuclei is therefore expected to be strongly anisotropic with a peak centered in the forward direction. 


\subsection{Imaging Trackers}

Nuclear emulsion are basically made of silver halide crystals embedded in a gelatin matrix. When ionizing particles pass through it, some of the halide crystals are modified in such a way that they are turned into grains of silver when a developing process is performed. The three-dimensional trajectory of passing through particles can be reconstructed with an optical microscope by connecting all the silver grains. NIT and U-NIT are a new generation of nuclear emulsion with a grain size of $48 \mathrm{~nm}$ and $18 \mathrm{~nm}$ respectively. NIT emulsion have a linear density of crystals of about 11 crystals/ $\mu \mathrm{m}[3]$ and they are made by the following nuclei (mass fraction in \%): $\mathrm{H}$ (1.6), C (10.1), N (2.7), O (7.4), S (0.3), Br (32.0), Ag (44.0), I (1.9). The total density is $3.2 \mathrm{~g} / \mathrm{cm}^{3}$. Taking in consideration resolution and linear density, a reconstruction of trajectories with path lengths shorter than $100 \mathrm{~nm}$ become possible using a proper microscope system. The presence of both light and heavy nuclei in the emulsion gel results in a good sensitivity to WIMPs with both light and heavy masses.

\subsection{Read-out system}

The expected WIMP signal will consist of short-path nuclear recoils, anisotropically distributed, over an isotropically distributed background. The read-out system has therefore to fulfill two main requirements: a fast and completely automated scanning system is needed to analyse the target volume over a time scale comparable with the exposure; the spatial resolution has to be improved by more than one order of magnitude compared to that achieved with standard emulsion films, reaching the challenging value of a few tens of nanometers, in order to ensure high efficiency and purity in the selection of signal candidates.

The analysis of NIT emulsions is performed with a two-step approach: first a fast scanning for the signal preselection, followed by a pin-point check of preselected candidates with nanometric resolution to further enhance the signal to noise ratio and perform very accurate measurements of the range and the recoil direction.

A fast scanning is performed using an improved version of the optical microscope used for the scanning of the OPERA films. In the last years an R\&D program was carried by INFN groups to improve the scanning performances [6], reaching a resolution improved by one order of magnitude and achieving a speed of almost $200 \mathrm{~cm}^{2} /$ hour [7]. A new system is being developed in Japan too aiming at increasing the scanning speed up to $5000 \mathrm{~cm}^{2} /$ hour [8].

The starting point of the emulsion scanning is the images acquisition of clusters made of dark grains at several depths across the emulsion plate thickness. Given the intrinsic resolution of the optical microscope $(\sim 200 \mathrm{~nm})$, the sequence of several grains making a track of a few hundred nanometers, appears as a single cluster. Therefore, the key element to distinguish clusters made of several grains from clusters made of a single grain produced by thermal excitation (fog) is the analysis of their shape. A cluster made of several grains indeed tends to have an elliptical shape with the major axis along the direction of the trajectory, while a cluster produced by a single grain tends to have a spherical shape. In order to simulate the effect of a WIMP-induced nuclear recoil and to measure the efficiency and the resolution of the new optical prototype, a test beam with low velocity ions was performed $[9,10]$.

The second phase is performed exploiting the resonance effect occurring when nanometric metal grains are dispersed in a dielectric medium [11]. The polarization dependence of the 
resonance frequencies strongly reflects the shape anisotropy and can be used to infer the presence of non-spherical nanometric silver grains.

NEWS will use this technology to retrieve track information in NIT emulsions beyond the optical resolution. Images of the same cluster taken with different polarization angles will show a displacement of its barycenter. The analysis of the displacements allows to distinguish clusters made of a single grain from those made of two (or more) grains. The evaluation of the position accuracy was performed by analysing images of single grains. The unprecedented accuracy of about $10 \mathrm{~nm}$ (Fig 1a) has been achieved in both coordinates, using an automatized optical microscope with rotating polarizer developed in the framework of NEWSdm collaboration (Fig $1 b)$.
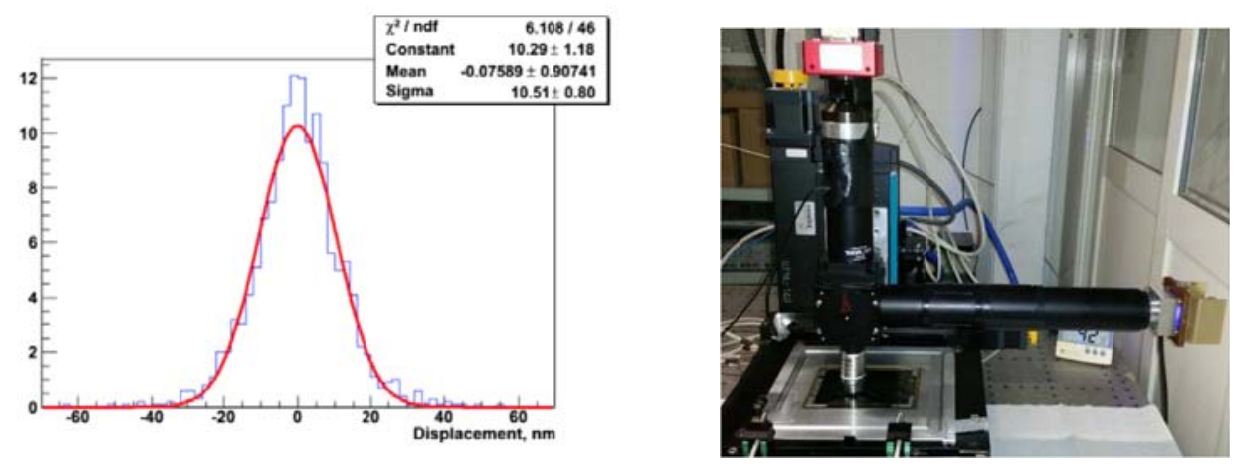

Figure 1. 1(a) Position accuracy of about $10 \mathrm{~nm}$ with resonant light scattering. 1(b) Microscope system with rotating polarizer.

\section{Background and sensitivity}

The main sources of background for dark matter search are $\alpha$ and $\beta$ particles, $\gamma$-rays and neutron induced recoils, while NIT are essentially not sensitive to minimum ionizing particles (MIP). The main sources of $\alpha$-particles are $U$ and Th radioactive chains and Radon. The $\alpha$-particles produced in those processes have energies of the order of $\mathrm{MeV}$ and their range in emulsion is of the order of tens of microns, by far longer than WIMP-induced nuclear recoils, $\alpha$-particles can therefore be identified and discarded in the emulsions by an upper cut on the track length. The $\beta$-rays produced in ${ }^{14} \mathrm{C}$ decay constitute a non-negligible contribution to the total background budget. This kind of background is anyway less critical for NIT emulsion with respect to other sources: electrons can be rejected by properly regulating the emulsion response, in terms of number of sensitized crystals per unit path length (i.e. the sensitivity), through a chemical treatment of the emulsion itself. Moreover possible improvements in the rejection power can be achieved exploiting the response of $\beta$-rays to the polarized light scattering or performing a cryogenic exposure and by exploiting the phonon effect. Neutron induced recoils are the main background source because they are not distinguishable from the expected WIMP signal, except for the isotropic angular distribution and for the typical track length, largely exceeding the range expected for WIMP-induced recoils. Three types of neutron sources affect underground experiments: radiogenic neutrons in the $\mathrm{MeV}$ range produced in $(\alpha, \mathrm{n})$ and spontaneous fission reactions in the detector due to its intrinsic radioactive contaminants, cosmogenic neutrons with energy spectrum extending to $\mathrm{GeV}$ energies induced by muons penetrating underground through the rock, neutrons induced by environmental radioactivity. While the external neutron flux can be reduced to a reasonable level with an appropriate 
shielding, the intrinsic emulsion radioactivity would be responsible of an irreducible neutron yield through $(\alpha, n)$ and ${ }^{238} \mathrm{U}$ spontaneous fission reaction. In order to estimate this contribution, the activities of $U$ and $T h$ in the emulsion components has been measured with the Inductively Coupled Plasma Mass Spectrometry (ICP-MS) and with the $\gamma$-spectrometry [12].

We plan to perform the first exposure with a target mass of $1 \mathrm{~kg}$ and the corresponding analysis of the data on a time scale of five years. During 2017 we performed a pilot exposure in order to confirm the estimations of the overall background budget of the experiment. A successful test was made in hall B of the Laboratori del Gran Sasso (LNGS) with 20 gr of NIT, shielded with lead and polypropylene blocks (Fig. 2). The next two years will be devoted to the construction of the infrastructures, the production of the emulsion target and of the detector shield. The exposure of $1 \mathrm{Kg}$ detector is foreseen by the beginning of 2020 and will last one year. We plan to get first analysis results by the end of 2021. In figure 3 the $90 \nmid \%$ C.L. upper limit, in case of null observation for an exposure of $1 \mathrm{~kg} x$ year of NIT emulsions, with a minimum detectable track length ranging from $200 \mathrm{~nm}$ down to $50 \mathrm{~nm}$ and in the hypothesis of zero background, is shown. Even not including the directionality discrimination of the signal and assuming to reach a negligible background level, such an experiment would cover a large part of the parameter space indicated by the DAMA/LIBRA results with a small $(1 \mathrm{~kg})$ detector mass, using a powerful and complementary approach.

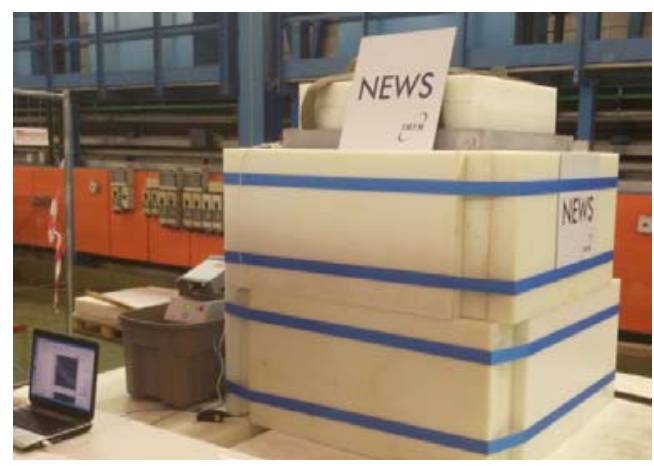

Figure 2: NEWSdm detector for pilot exposure

in hall B of the Laboratori Nazionali del Gran Sasso

\section{Conclusion}

NEWS is meant to be the first experiment with a solid target for directional dark matter searches: the use of a nuclear emulsion based detector, acting both as target and tracking device, would allow to extend dark matter searches beyond the neutrino floor and provide an unambiguous signature of the detection of Galactic dark matter. The novel emulsion technology, based on the use of nuclear emulsion with nanometric $\mathrm{AgBr}$ crystals (NIT), makes it possible to record the sub-micrometric tracks produced by the WIMP scattering off a target nucleus. The presence, in the emulsion components, of light and heavy nuclei results in an enhanced sensitivity to both light and heavy WIMP masses. The final signal confirmation is obtained with powerful optical microscope equipped with a light polarizer: exploiting the different response of non spherical grain clusters to different polarization angles, the unprecedented spatial resolution of $10 \mathrm{~nm}$ is obtained. This resolution allows to resolve grains belonging to a few hundred of nanometer long tracks thus providing the final signal confirmation with a very high signal to 
noise ratio. We plan to perform a pilot experiment with a $1 \mathrm{~kg}$ mass target on a time scale of five years: even using a rather small detector mass we would be able to explore the region indicated by the DAMA experiment with a powerful and complementary approach. The actual intrinsic radioactive level allows to scale the target mass and exposure time up to one order of magnitude. A careful selection of the emulsion components and a better control of their production could further increase the radiopurity, thus allowing larger detector mass. The reduction of the fog density and further developments of the optical microscopy with polarized light would allow to reduce the detection threshold down to $50 \mathrm{~nm}$. Improvements both in the mechanics (use of piezoelectric-driven objective) and in the image acquisition (use of multiple image sensors) envisage the possibility to analyse with such a resolution a volume of $100 \mathrm{~kg}$ or larger.

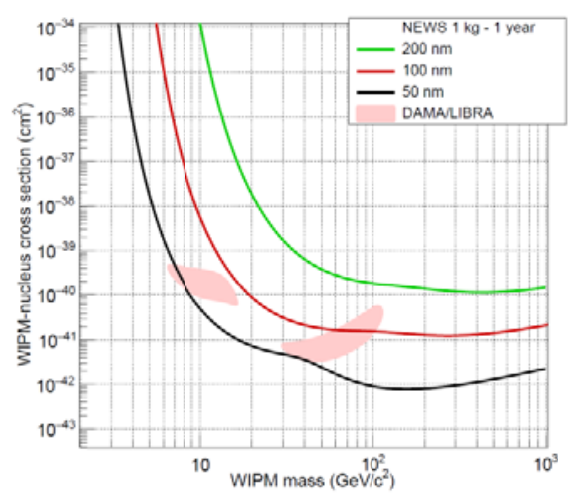

Figure 3: The 90\% C.L. upper limits for a NIT detector with an exposure of $1 \mathrm{~kg}$ x year, a threshold ranging from $200 \mathrm{~nm}$ down to $50 \mathrm{~nm}$, in the zero background hypothesis. The directionality information is not included.

\section{References}

[1] J.B.R. Battat et al., Phys. Rep 662 (2016) 1

[2] A Letter of Intent - NEWSdm Collaboration, arXiv:1604.04199, LNGS-LOI 48/15

[3] M. Natsume et al., Nucl. Instr. Meth. A575 (2007) 439

[4] T. Naka et al., Nucl. Instrum. Meth. A718 (2013) 519

[5] A. Agafonova et al. (OPERA Collaboration), JINST 4 (2009) P04018.

[6] L. Arrabito et al., Nucl. Instr. Meth. A568 (2006) 578.

[7] A. Alexandrov, V. Tioukov, M. Vladymyrov, JINST 9 (2014) C02034

[8] K. Morishima and T. Nakano, JINST (2010) P04011

[9] T. Naka et al., EAS Publ. Ser. 53 (2012) 51

[10] M. Kimura and T. Naka, Nucl. Instrum. Meth, A680 (2012) 12

[11] H. Tamaru et al., App. Phys. Lett. 80 (2002) 1826

[12] A. Alexandrov et al., Astropart. Phys, 80 (2016) 16. 